\title{
Effect of Lignin and CNTs on the properties of melt-spun polymeric fibers
}

\author{
Panagiotis Goulis ${ }^{1}$, Ioannis A. Kartsonakis ${ }^{1}$, Konstantinos Mpalias ${ }^{1}$ and Costas A. \\ Charitidis ${ }^{1, *}$ \\ 1,* National Technical University of Athens, Department of Chemical Engineering, RNANO Lab - \\ Research Unit of Advanced, Composite, Nanomaterials and Nanotechnology, 9 Heroon Polytechneiou \\ st., Zografos Athens, GR-15773
}

\begin{abstract}
The quality of high density polyethylene (HDPE) - lignin spun fibers depends on the spinning method and the chemical modification of the raw material. On the other hand, nylon-type polymers are widely known for their simple experimental synthesis and use. The goal of this study was the fabrication of composites containing lignin, nylon and multi-walled carbon nanotubes (MWCNTs) based fibers with enhanced mechanical properties. Therefore, a nylon-type polymer was synthesized, using diethylenetriamine and sebacoyl chloride, and was conjugated with lignin as well as MWCNTs with the aim of determining the materials' compatibility, differences in chemical behavior as well as their ability to be melt spun and be further processed. The materials were compounded with HDPE and melt spun, producing fibers that were characterized in respect of their morphology, structural, chemical and elemental composition. Finally, the HDPE fibers were used for the production of one dimensional composites inside dumb bell type sacrificial templates with an epoxy resin solution. The composites, which contained fibers enriched with oxidized lignin and MWCNTs, presented higher elastic modulus and ultimate tensile strength than the rest of the composites. This is a novel attempt, presenting an innovation concerning durable fibers with low cost materials and procedures.
\end{abstract}

\section{Introduction}

Nowadays a lot of capital is being invested in the manufacturing of fibers. The need for this cost to be reduced is desirable, especially when large scale production is necessary to support the industries. Most precursor materials are very expensive, so as the need for low cost raw materials increases, the interest is focused on natural, abundant polymers and on green processes in general $[1,2]$. Currently, lignin is being investigated as to the amount of its potential contribution to the future of fibers. Fiber composites are indispensable high strength materials to the automotive industry, the aerospace industry etc. [3, 4]. Although, lignin was on the scope of polymer scientists for years, its implementation into polymer science and engineering is often hindered by its structure, which is highly dependent on the extraction

\footnotetext{
*Costas A. Charitidis: charitidis@,chemeng.ntua.gr
} 
procedure and the type of plant selected $[5,6]$. On the other hand, nylon is widely used at a galore of everyday applications such as polymeric films, plastic bags and other products [79]. Several major industries over the world, such as the automobile industry, are constantly searching for materials with desirable and favorable properties in order to enhance their products and be more competitive in a global scale. Efforts are being made towards the direction of reducing the production cost of innovative and smart materials, by synthesizing composites that combine the useful properties of two or more compounds $[10,11]$. Also, the procedure of cutting down on the production costs of raw materials or on the costs of their further processing and development has always been on the scope of researchers and manufacturers [12,13].

In this study, fibers have been successfully melt-spun, using low cost raw materials, such as commercial high-density polyethylene (HDPE), a synthesized nylon-type polymer, softwood kraft lignin (SKL) and multi-walled carbon nanotubes (MWCNT) [14]. All the samples have been characterized via scanning electron microscopy (SEM), Fourier transformed infrared spectroscopy (FT-IR), tensile testing, thermal analysis and x-ray tomography (Micro-CT) and their properties were compared to one another in order to determine the role of each compound on the final product. Furthermore, these fibers were molded together in combination with epoxy resin, in order to form epoxy composites enriched with fibers. These samples were subjected to tensile strength in order for their tensile strength to be determined, with the aspect of being used as reinforcing materials $[15,16]$. This is a novel approach, which complies with the motivation of this work to fabricate durable fibers along with the minimum cost and simple experimental methods.

\section{Materials and Methods}

\subsection{Materials}

All chemicals were of analytical reagent grade. High-density polyethylene (HDPE, Sigma Aldrich, St. Louis, Missouri, USA, Mw 125,000), hydrochloric acid (HCl 37\%, Fisher Chemicals, Waltham, Massachusetts, USA), sebacoyl chloride (Acros Organics, Geel, Belgium), diethylene triamine $\left(\mathrm{CHCl}_{3}\right.$, Fisher Chemicals, Waltham, Massachusetts, USA), camphor (96\% purity in weight, Aldrich)as carbon precursor and ferrocene $(98 \%$ purity in weight, Aldrich), acetylene (Air Liquide), $\mathrm{Fe}\left(\mathrm{NO}_{3}\right)_{3} .9 \mathrm{H}_{2} \mathrm{O}$ (Aldrich, St. Louis, USA), zeolite Y (Alfa Aesar; particle size $\sim 1 \mu \mathrm{m}$; specific surface area $975 \mathrm{~m}^{2} / \mathrm{g}$ ), bisphenol-A/F monofunctional RD (ER 1042-7, Ipox Chemicals GmbH, Laupheim, Germany), modified cycloaliphatic amine (EH 2117, Ipox Chemicals GmbH, Laupheim, Germany) and potassium dichromate (Fisher Chemicals, Waltham, Massachusetts, USA) were used as received without further purification. Softwood kraft lignin (SKL, Westvaco Corp., Indulin AT, MWV, Norcross, Georgia, USA) was treated with $\mathrm{HCl} 0.1 \mathrm{M}$ prior to its use. All meltspinning blends were dried in a vacuum oven for $24 \mathrm{~h}$ prior to their extrusion.

\subsection{Synthesis}

The synthesis of MWCNTs was performed according to our previous work [17] via chemical vapor deposition method using a thermal reactor that consists of an horizontal quartz tube housed in a three-zone cylindrical furnace. Briefly, a pyrex flask containing the reagent mixture, which was composed by camphor as carbon precursor and ferrocene as catalyst compound in a 20/1 mass ratio, was connected to the tube nearby the nitrogen inlet. A heater plate below the flask achieved heating and sublimation of the reactants. Nitrogen gas flow 
carried the gas mixture of precursors towards the center of the furnace, where pyrolysis of the gases took place at $850^{\circ} \mathrm{C}$ resulting in the formation and deposition of MWCNTs carpets on silicon wafers substrates. When the reaction was completed, the raw products were cooled down to room temperature in $\mathrm{N}_{2}$ atmosphere. After the synthesis, the raw products were milled and exposed at atmospheric air flow at $400^{\circ} \mathrm{C}$ for $1 \mathrm{~h}$, aiming at the removing of the amorphous carbon. Afterwards, they were purified with constant boiling of $5 \mathrm{M} \mathrm{HCl}$ in a Soxhlet extractor in order to remove the remaining metal particles. Finally, the purified MWCNTs were washed with distilled water and dried in an oven. To activate the MWCNTs surface with $-\mathrm{COOH}$ groups, an acid solution mixture of $6 \mathrm{M} \mathrm{HNO}_{3}: \mathrm{H}_{2} \mathrm{SO}_{4}$ 1:3 was used. Then, the MWCNT/acid mixture $(0.15 \mathrm{~g} \mathrm{CNTs} / 10 \mathrm{ml}$ acid solution) was stirred for $48 \mathrm{~h}$ at $80^{\circ} \mathrm{C}$. The suspension was filtered and the black powder deposited on the filter is washed with distilled water, then with ethanol and acetone and dried in oven.

Furthermore, in a $600 \mathrm{ml}$ glass beaker, $1.7 \mathrm{~g}$ of potassium dichromate $\left(\mathrm{K}_{2} \mathrm{Cr}_{2} \mathrm{O}_{7}\right)$ were dissolved in $200 \mathrm{ml}$ of distilled and deionized water. In another glass beaker of $1,000 \mathrm{ml}, 20 \mathrm{~g}$ of washed lignin (SKL) were dissolved in $140 \mathrm{ml}$ of acetone. Then, the first solution was added to the second one and the temperature was set to $60^{\circ} \mathrm{c}$ for $2 \mathrm{~h}$, in order for the lignin to be oxidized (ox.SKL). After that, $20 \mathrm{ml}$ of sebacoyl chloride (SEBAC) and $30 \mathrm{ml}$ of diethylenetriamine (DETA) was added to the mixture. The addition of sebacoyl chloride to the blend is a very exothermic reaction, which is done under vigorous manual stirring. After 2 more hours of stirring, the product was dried in an oven at $80^{\circ} \mathrm{C}$ and then pulverized $[P(D E T A$-alt-SEBAC)-ox.SKL]. Chemical equations of lignin's oxidation and the polymerization reaction between diethylenetriamine and sebacoyl chloride are depicted in Figure 1 and Figure 2, respectively, whereas, the chemical equation of the reaction between oxidized lignin and the nylon polymer is illustrated in Figure 3. Finally, the same experiments were repeated adding the synthesized MWCNTs, obtaining the corresponding product $P(D E T A$-alt-SEBAC)-ox.SKL-MWCNTS.

(1)

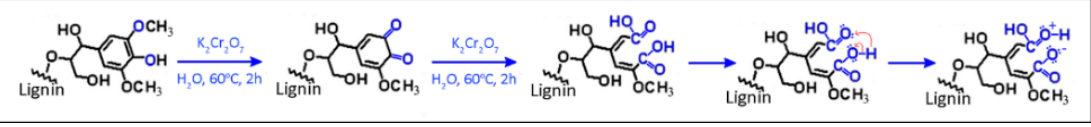

Fig. 1. Oxidation of lignin

(2) $\mathrm{H}_{2} \mathrm{~N} \sim \mathrm{N}^{\mathrm{N}} \longrightarrow \mathrm{NH}_{2} \leftrightarrows \underset{\mathrm{H}}{\mathrm{N}} \sim \mathrm{N}^{\mathrm{H}} \sim_{\mathrm{NH}_{2}}+\mathrm{H}^{+}$

(3)

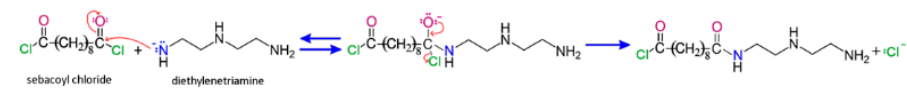

(4)

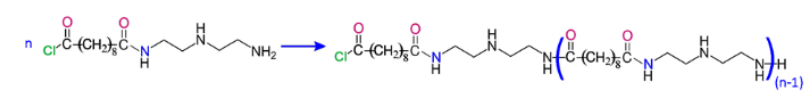

Fig. 2. Reaction between diethylenetriamine and sebacoyl chloride

\subsection{Melt Spinning}

The abovementioned materials were melt spun, using a melt spinning apparatus, while mixed with the commercial polymer HDPE in the form of pellets. Commercial HDPE was purchased in pellets and were of relatively low molecular weight. The SKL was purified prior its use with $\mathrm{HCl} \mathrm{0.1} \mathrm{M.} \mathrm{Four} \mathrm{different} \mathrm{fibers} \mathrm{were} \mathrm{produced} \mathrm{as} \mathrm{it} \mathrm{is} \mathrm{tabulated} \mathrm{in} \mathrm{Table} \mathbf{1}$. 


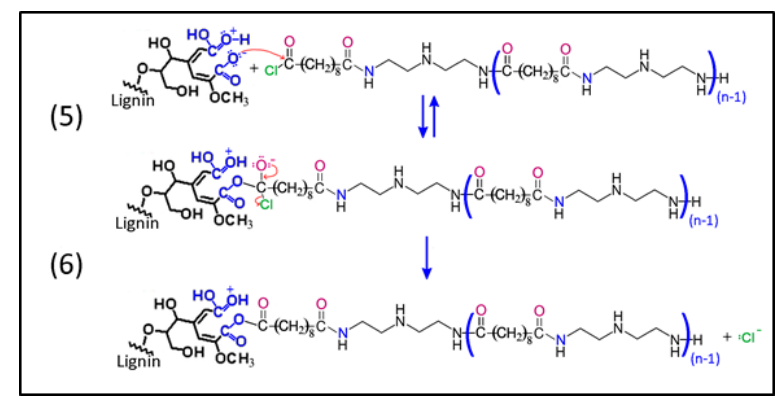

Fig. 3. Reaction between oxidized lignin and nylon polymer

Table 1. Compositions of the produced fibers.

\begin{tabular}{ccccc}
\hline Fibrous Materials & $\begin{array}{c}\text { HDPE } \\
\text { wt. } \%\end{array}$ & $\begin{array}{c}\text { ox.SKL } \\
\text { wt. } \%\end{array}$ & $\begin{array}{c}\text { P(DETA-alt- } \\
\text { SEBAC)- } \\
\text { ox.SKL wt. } \%\end{array}$ & $\begin{array}{c}\text { P(DETA-alt- } \\
\text { SEBAC)-ox.SKL- } \\
\text { MWCNs wt. } \%\end{array}$ \\
\hline HDPE & 100 & 0 & 0 & 0 \\
\hline HDPE/ ox.SKL & 75 & 25 & 0 & 0 \\
\hline $\begin{array}{c}\text { HDPE/ P(DETA-alt- } \\
\text { SEBAC)-ox.SKL }\end{array}$ & 75 & 0 & 25 & 25 \\
\hline $\begin{array}{c}\text { HDPE/ P(DETA-alt- } \\
\text { SEBAC)-ox.SKL- } \\
\text { MWCNTs }\end{array}$ & 75 & 0 & 0 & 0 \\
\hline
\end{tabular}

\section{Results and Discussion}

The oxidized lignin (ox.SKL) contains numerous COO-groups, which can react with the hydrogen atoms bonded to the nitrogens and the atom of chloride at the end of the polymeric chains of the nylon-type polymer (Figure 3). The oxidized lignin forms hydrogen bonds with the nitrogen atoms of the nylon polymer, thus making the conjugation of lignin to the polymer structure stronger and feasible. It should be mentioned that lignin contains numerous $\mathrm{C}-\mathrm{OH}$ bonds and the oxidation is not a one-way reaction. Therefore, not all of lignin's hydroxyl groups become oxidized. Furthermore, due to the long polymer chains and the steric hindrance of the complex lignin molecules and the nylon-type polymer, it is expected that not all ${\mathrm{C}-\mathrm{O}^{-}}^{-}$oxidized lignin groups have reacted to form the final product. Nevertheless, the reactants that eventually reacted, formed the final product which is different in color, thermal behavior and texture.

The maximum mass of the synthesized compound (with lignin or not) that could be achieved in the blend was $25 \mathrm{wt} . \%$ (Table 1). Beyond this percentage the blends were neither desirably treatable, nor were they spinnable. The HDPE commercial polymer used, was selected due to its spinning ability, known from previous experiments. It should be noticed that the fibers which contained no lignin, were thicker macroscopically with a lot of agglomerates on the fiber's surface. This phenomenon indicates that these blends do not have sufficient spinning ability, neither are they compatible with the commercial polymers. On the other hand, the fibers with lignin had a reduced amount of agglomerates and their surface was smoother and thinner macroscopically. Furthermore, the best results were observed for 
the fibers containing oxidized lignin. These samples presented close to none agglomerates and an even smoother surface. Additionally, there was a gradation of colors, going from the plain nylon polymer fibers to those containing oxidized lignin. The lignin enriched fibers had a deep brown color.

\subsection{Characterization}

The morphology as well as the elemental composition of the synthesized fibers, were studied via a Hitachi Tabletop Microscope TM3030 Scanning Electron Microscopy and with an Ultra-High-Resolution Scanning Electron Microscopy (UHR-SEM) using NOVA NANOSEM 230 (FEI Company). TR-FTIR analysis was conducted on a Cary 630 spectrometer (Agilent). TGA/DSC analysis was conducted on a simultaneous TG-DTA/DSC Apparatus (STA 449 F5 Jupiter). The internal structural information of the samples was observed and collected by the compact desk-top Bruker micro-CT, 3D X-ray scan system, SkyScan 1272. The tensile strength at the breaking point of the fiber was determined according to the scale of the dynamometer of the MPM-10 tensile test machine, based on the standards ISO 11566:1996 and ASTM D 3379-89. The mechanical properties of the composites were investigated by tensile tests which were performed using a Zwicki 1120 apparatus, fitted with a $100 \mathrm{~N}$ dynamometer at room temperature.

\subsubsection{Scanning Electron Microscopy}

Concerning SEM analysis, as it is evidenced from the Figure 4, smaller diameters are observed for the samples without ox.SKL and MWCNTs. The addition of oxidized lignin causes an increase of the fiber diameter of the plain $H D P E$. Therefore, the arithmetical values of the fiber diameters are increased as we move from plain $H D P E$ fibers to $H D P E / P(D E T A$ alt-SEBAC)-ox.SKL fibers and even more to HDPE/P(DETA-alt-SEBAC)-ox.SKL-MWCNTS fibers. This fact shows that the addition of ox.SKL and MWCNTs in the blends caused agglomerations and miscibility problems with HDPE. Although MWCNTs caused an overall increase in the diameters, they contributed to the higher strength and stress tolerance of the materials (tensile strength).



Fig. 4. SEM images of the a) HDPE, b) HDPE/ ox.SKL, c) HDPE/ P(DETA-alt-SEBAC)-ox.SKL and d) HDPE/ P(DETA-alt-SEBAC)-ox.SKL-MWCNTs fibers. 


\subsubsection{Thermal Analysis}

Concerning Figure 5, it can be seen that the fibers without MWCNTs degrade at a relatively low temperature range, even when the temperature remains stable for a substancial amount of time. However, as far as the MWCNTs enriched fibers are regarded, they show an enhanced thermal behavior. More specifically, an abrupt mass loss of about $60 \%$ of the initial mass is observed until the temperature reaches $150^{\circ} \mathrm{C}$. Then, the degradation continues gradually at a controlled and smooth manner. Even when the temperature reaches $900^{\circ} \mathrm{C}$, the fibers seem to retain more than $20 \%$ of their initial weight.
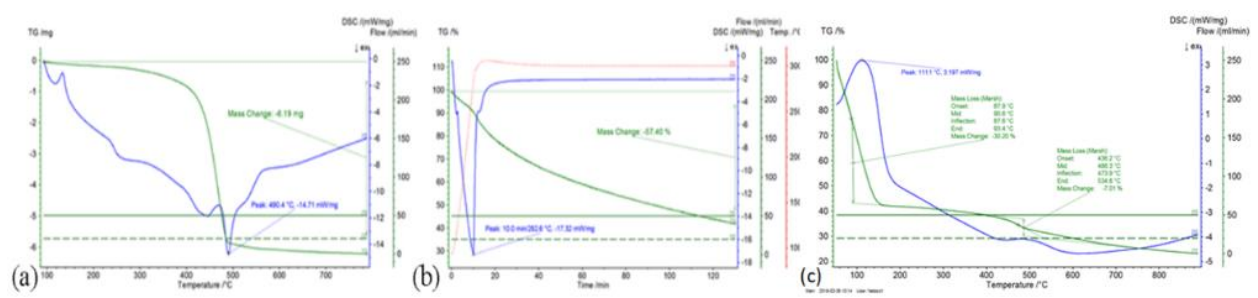

Fig. 5. a) Dynamic and b) isothermal TGA/DSC graphs for HDPE/ P(DETA-alt-SEBAC)-ox.SKL fiber. c) TGA/DSC diagrams for the HDPE/ P(DETA-alt-SEBAC)-ox.SKL-MWCNTs fiber.

\subsubsection{Micro-CT X-Ray Tomography}

Regarding the oxidized lignin fiber samples, it may be mentioned that the presence of MWCNTs had a substantial impact to the fiber properties, as it is evidenced via Micro-CT analysis. After the addition of MWCNTs, the structure thickness is increased, due to the more compact form, that MWCNTs induce to the fibers. To this phenomenon is also ascribed the higher value of the structure linear density. Moreover, the total porosity is also reduced, a fact that shows that MWCNTs helped to reduce the inner pores of the material, via their complicated structure. The results of this comparison are presented in Table 2 and the arrangement of the fibers into the epoxy resin matrix is demonstrated in Figure 6.

Table 2. Micro-CT arithmetical results for the oxidized lignin fiber epoxy composites

\begin{tabular}{ccc}
\hline Parameters & $\begin{array}{c}\text { HDPE/P(DETA-alt- } \\
\text { SEBAC)-ox.SKL }\end{array}$ & $\begin{array}{c}\text { HDPE/P(DETA-alt- } \\
\text { SEBAC) }\end{array}$ \\
\hline Object Surface to Volume ratio & 19.94 & 19.96 \\
Object Surface Density $\left(\mathrm{mm}^{-1}\right)$ & 19.78 & 19.80 \\
Structure Thickness $(\mathrm{mm})$ & 0.168 & 0.579 \\
Structure Separation (mm) & 0.023 & 0.015 \\
Structure Linear Density (mm $\left.{ }^{-1}\right)$ & 5.888 & 8.954 \\
Degree of Anisotropy & 4.418 & 2.389 \\
Fractal Dimension & 1.969 & 1.911 \\
Total Porosity (\%) & 0.794 & 0.201 \\
\hline
\end{tabular}




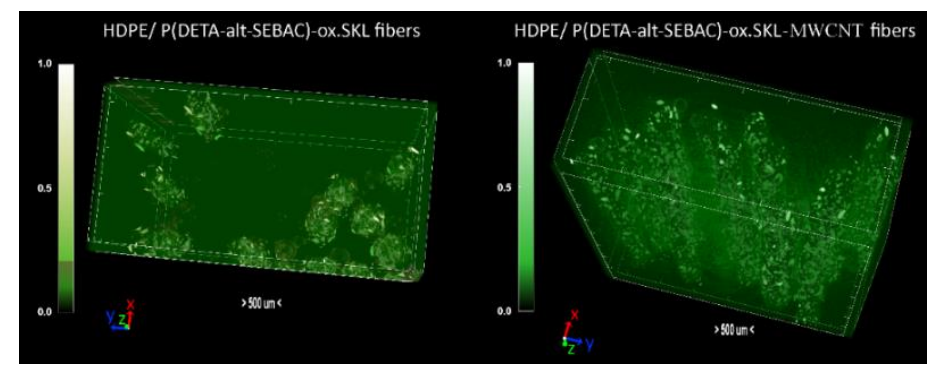

Fig. 6. Arrangement of fibers into the epoxy resin matrix via Micro-CT visualization.

\subsubsection{Tensile Strength}

The produced fibers were used for the production of one dimensional composites. The polymer coated fibers were placed inside dumb bell type sacrificial templates and then an epoxy resin solution consisting of Bisphenol-A/F monofunctional RD (ER 1042-7) and modified cycloaliphatic amine (EH 2117) was added to each template. The dimensions of the templates were selected in agreement with ASTM D638-14. The dogbone specimens underwent tensile strength tests in order to determine their elastic modulus and mechanical durability. It was evidenced via tensile tests, that these composites had advanced mechanical properties, due to the addition of the fibers in the polymeric matrix. More specifically, the composites, which contained fibers enriched with MWCNTs and oxidized lignin, presented higher elastic modulus and could withstand higher force of tensile strength than the other composites. The comparative tensile stress graphs for the four different composite fiber materials are demonstrated in Figure 7. It can be clearly noticed that composite including fibers, which contained MWCNTs, could withstand higher stress, before eventually breaking.

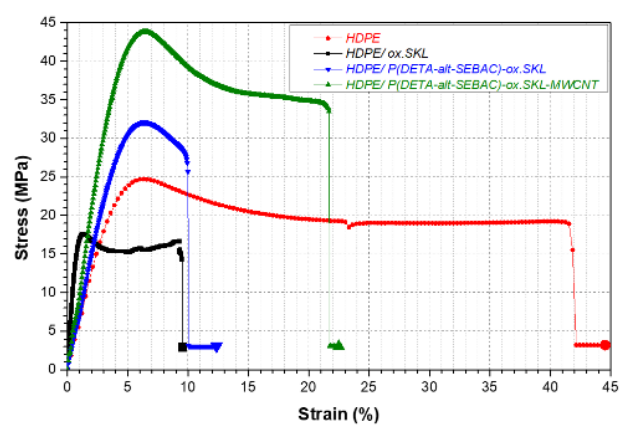

Fig. 7. The stress vs strain diagrammes of the dumbbell fiber - epoxy composites including HDPE based fibers.

\section{Conclusions}

In this comparative study, different materials were synthesized in order to investigate the role of lignin and MWCNTs to a nylon polymer's properties and spinning ability. So, these samples were mixed with the commercial spinnable polymer HDPE and were melt spun to 
produce fibers, which were characterized via SEM analysis, TGA/DSC analysis and Micro$\mathrm{CT}$ analysis. Comparison of these results showed that lignin enhanced the properties of the nylon polymer, but oxidized lignin in combination with CNTs presented even better properties. After that, the fibers were molded together with epoxy resin in the appropriate molding templates in order to perform tensile strength tests, so as for the physical properties of these polymeric fibers to be determined and examine as to what extent these materials could function as reinforcing intermediates. It is concluded that lignin improves the fiber texture and mechanical/thermal performance compared to the plain nylon polymer, but oxidized lignin and MWCNTs improve the materials even more. The innovation of this study lies in the combination of MWCNTs and lignin along with a commercial and a chemically synthesized polymer to fabricate durable low-cost fibers via a simple method, so as to enhance the tensile properties of composites.

This work was supported by the EU FP7 Project "Functionalized Innovative Carbon Fibres Developed from Novel Precursors with Cost Efficiency and Tailored Properties" (FIBRALSPEC) under Grant Agreement no. 604248 .

\section{References}

1. N. Meek, D. Penumadu, O. Hosseinaei, D. Harper, S. Young, T. Rials, Comp. Sc. Tech. 137, 60-68 (2016)

2. A. Duval, M. Lawoko, Reac. Funct. Pol. 85, 78-96 (2014).

3. A.R. Jabur, Int. J. Hydr. En. (2017)

4. R. Zhu, V. Yadama, H. Liu, R.J.T. Lin, D.P. Harper, Comp. P. A: Appl. Sc. Man. 97, 111-119 (2017)

5. E. Koivuranta, M. Hietala, A. Ämmälä, K. Oksman, M. Illikainen, Comp. P. A: Appl. Sc. Man. 101, 265-272 (2017)

6. P. Kainourgios, I.A. Kartsonakis, D.A. Dragatogiannis, E.P. Koumoulos, P. Goulis, C.A. Charitidis, Appl. Surf. Sc. 416, 593-604 (2017)

7. L. Dehne, C. Vila Babarro, B. Saake, K.U. Schwarz, Ind. Cr. Prod. 86, 320-328 (2016)

8. O. Gordobil, I. Egüés, R. Llano-Ponte, J. Labidi, Pol. Degr. Stab. 108, 330-338 (2014)

9. C.D.A.C. Erbetta, G.F. Manoel, A.P.L.R. Oliveira, M.E.S.R.e. Silva, R.F.S. Freitas, R.G. Sousa, Mat. Sc. Applic. 05, 923-931 (2014)

10. P. Goulis, G. Konstantopoulos, I.A. Kartsonakis, K. Mpalias, S. Anagnou, D. Dragatogiannis, C.A. Charitidis, C 3, 35 (2017)

11. H. Mainka, O. Täger, E. Körner, L. Hilfert, S. Busse, F.T. Edelmann, A.S. Herrmann, J. Mat. Res. Tech. 4, 283-296 (2015)

12. C.I. Simionescu, V. Rusan, M.M. Macoveanu, G. Cazacu, R. Lipsa, C. Vasile, A. Stoleriu, A. Ioanid, Comp. Sc. Tech. 48, 317-323 (1993)

13. D. Kun, B. Pukánszky, Eur. Pol. J. (2017)

14. S. Laurichesse, L. Avérous, Prog. Pol. Sc. 39, 1266-1290 (2014)

15. W. Jin, D. Shen, Q. Liu, R. Xiao, Pol. Degr. Stab. 133, 65-74 (2016)

16. J. Zhao, W. Xiuwen, J. Hu, Q. Liu, D. Shen, R. Xiao, Pol. Degr. Stab. 108, 133-138 (2014)

17. A.-F.A. Trompeta, E.P. Koumoulos, I.A. Kartsonakis, C.A. Charitidis, Man. Rev. 4, 7 (2017). 